\title{
Steckt hinter dem Zufallsbefund an der Nebenniere ein Karzinom?
}

\author{
Eine zufällig entdeckte Raumforderung an der Nebenniere sollte abgeklärt werden, auch \\ wenn sie in den meisten Fällen harmlos ist. Eine neue Praxisleitlinie gibt vor, wie man mit \\ relativ unkomplizierten Untersuchungen die beiden wichtigsten Fragen beantworten kann: \\ Ist der Tumor bösartig? Und: Ist er hormonell aktiv?
}

\begin{abstract}
Ein Tumor an der Nebenniere wird, wenn überhaupt, in der Regel zufällig bei einer bildgebenden Untersuchung entdeckt. Bei über 50-Jährigen liegt die Inzidenz eines solchen Inzidentaloms bei 3\%, von den über 80 -Jährigen hat es bereits jeder Zehnte. Nach Prof. Martin Fassnacht, Würzburg, handelt es sich in $80 \%$ der Fälle um ein benignes, hormoninaktives Geschehen, das man „am besten in Ruhe lässt“. Das Problem ist aber, dass man zunächst nicht wissen kann, ob nicht doch ein Karzinom dahintersteckt. Der Begriff „Inzidentalom“ bedeutet nicht, dass der Tumor in jedem Fall benigne ist.
\end{abstract}

\section{Malignität und Hormonexzess abklären!}

Um Fälle wie den im Kasten geschilderten zu verhindern, hat die European Society for Endocrinology (ESE) unter Federführung von Fassnacht eine Leitlinie entwickelt [1]. Danach sind für die Diagnose primär zwei Fragen relevant:

- Ist die Raumforderung potenziell maligne?

- Liegt ein relevanter Hormonexzess vor?

Das „mit Abstand einfachste und beste“ Verfahren zur Abklärung der Malignität ist laut Fassnacht die native CT: Der Radiologe muss dabei die sog. HounsfieldEinheiten bestimmen, ein Maß für die Abschwächung von Röntgenstrahlen. Liegt dieser Wert unter 10, kann ein malignes Geschehen praktisch ausgeschlossen werden. Eine weitere Möglichkeit ist die CT mit zehnminütigem Kontrastmit-
tel-Washout. Auch dieses Verfahren ist, so Fassnacht durch „sehr gute Daten“ gestützt. Für Benignität spricht ein Washout von über 60\% (absoluter Washout) bzw. über 40\% (relativer Washout)

Um die Frage nach dem Hormonexzess zu klären, seien Anamnese und körperliche Untersuchung Voraussetzung. Danach kommen zwei Tests infrage:

- der Test auf Plasma-Metanephrine zum Ausschluss eines Phäochromozytoms sowie

- der 1-mg-Dexamethason-Hemmtest, mit dem sich ein Hyperkortisolis-

mus nachweisen lässt.

Bei gleichzeitig vorliegender Hypertonie oder Hypokaliämie sei es noch ratsam, den Aldosteron/Renin-Quotienten zu erheben. Dieser gibt ggf. Aufschluss über einen Hyperaldosteronismus.

\section{Ein Fall aus der Klinik}

Eine 33-jährige Patientin hatte wegen Endometriosebeschwerden eine MRT erhalten. Diese zeigte eine $2,5 \mathrm{~cm}$ große Raumforderung an der linken Nebenniere. Aus der Radiologieabteilung kam der Befund: „Entspricht am ehesten einem Inzidentalom." Routinemäßig wurde die Patientin nach sechs Monaten zur Verlaufskontrolle einbestellt, dabei stellte sich heraus, dass der Tumor auf $9 \mathrm{~cm}$ gewachsen war und bereits in die Leber metastasiert hatte. Zusätzlich hatte sich ein Tumorthrombus in der Vena cava gebildet. 13 Monate nach der Karzinomdiagnose war die Patientin tot.

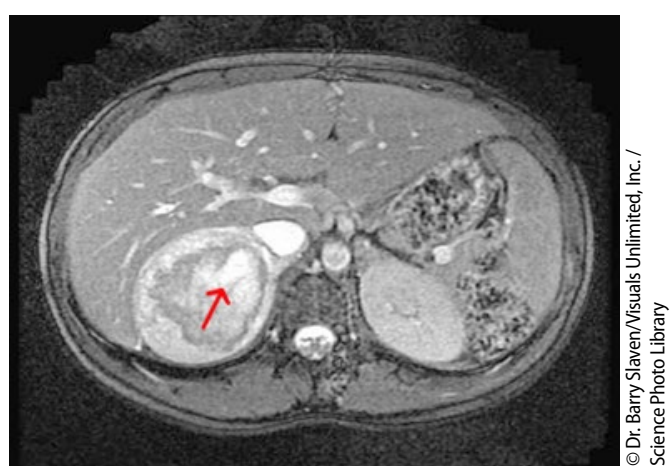

Hormon-produzierender Nebennierentumor (Pfeil): Phäochromozytom.

\section{Oft kein Follow-up erforderlich}

Für alle Läsionen, die sich in den genannten Tests als nicht maligne und nicht funktionell erwiesen haben, ist kein Follow-up mehr nötig. Alle anderen Fälle sollten laut Leitlinie in einem multidisziplinären Team besprochen werden:

- Beim Nebennierenadenom mit autonomer Kortisolsekretion (1-mgDexamethason-Test $>1,8 \mu \mathrm{g} / \mathrm{dl}$ ) werden die entsprechenden Komorbiditäten, u. a. arterielle Hypertonie, Typ-2-Diabetes, Dyslipidämie oder Osteoporose, behandelt.

- Bei klinisch relevantem Hormonexzess oder Malignitätsnachweis ist eine Op. indiziert.

- Bei weiterhin unklarem Befund ist die Entscheidung zwischen Op. oder Nachbeobachtung zu treffen. Dr. Elke Oberhofer

- Internisten-Update, 18./19. November 2016 in München 1. Fassnacht M et al. Eur J Endocrinol 2016;175:G1-34 